\title{
Remote Sensing Satellite Image Processing Techniques for Image Classification: A Comprehensive Survey
}

\author{
Sowmya D. R. \\ Research Scholar \\ Department of CSE, \\ University Visvesvaraya \\ College of Engineering, \\ Bangalore University, \\ Bengaluru, India
}

\author{
P. Deepa Shenoy \\ Professor, \\ Department of CSE, \\ University Visvesvaraya \\ College of Engineering, \\ Bangalore University, \\ Bengaluru, India
}

\author{
Venugopal K. R. \\ Professor and principal \\ Department of CSE, \\ University Visvesvaraya \\ College of Engineering, \\ Bangalore University, \\ Bengaluru, India
}

\begin{abstract}
This paper is a brief survey of advance technological aspects of Digital Image Processing which are applied to remote sensing images obtained from various satellite sensors. In remote sensing, the image processing techniques can be categories in to four main processing stages: Image preprocessing, Enhancement, Transformation and Classification. Image pre-processing is the initial processing which deals with correcting radiometric distortions, atmospheric distortion and geometric distortions present in the raw image data. Enhancement techniques are applied to preprocessed data in order to effectively display the image for visual interpretation. It includes techniques to effectively distinguish surface features for visual interpretation. Transformation aims to identify particular feature of earth's surface and classification is a process of grouping the pixels, that produces effective thematic map of particular land use and land cover.
\end{abstract}

\section{Keywords}

Classification, Image, Enhancement, Remote Sensing, Resolution, Satellite Sensors

\section{INTRODUCTION}

Remote sensing is the process of gathering data about the earth's surface without being in contact with it. This is process is done by sensing and recording emitted or reflected energy and then processing, analyzing and applying that information. Remote sensing process includes the illumination or energy source which passes through the atmosphere and interacts with the target; the electromagnetic energy emitted or scattered from the target is collected and recorded by the satellite sensors is transmitted in electronic form to a receiving and processing station where the data is processed into an image. The processed image is interpreted visually or electronically or digitally to extract the information about the illuminated target. Remote sensing systems which measure reflected energy are called passive sensors, which can be used only to detect energy in the present of naturally occurring energy. This can take place only during the time when the sun is illuminating the earth.

An active sensor provides its own energy source for illumination. The sensors emit radiation which is directed towards the target to be investigated; these sensors obtain the information regardless of the time of day. In order to capture the earth's surface the sensors must be paced in a proper platform. Before it was ground-based and aircrafts platforms, nowadays satellite near-polar orbits platform provides a great contribution to remote sensing imagery.

Motivation : The Multispectral satellite sensor provides digital raster images, that allow us to apply Digital Image Processing (DIP) techniques to develop thematic maps of landuse/landcover classes which are essential in many remote sensing applications like forestry, agriculture, environmental studies, weather forecasting, ocean studies, archeological studies etc.

Contribution: In this paper various advanced image processing techniques to convert raw satellite imagery into fine data obtained from different spatial, spectral and temporal resolutions from microwave to ultraviolet bands are discussed.

Organization: The paper is organized as follows; Section I deals with various resolutions of satellite sensor. Section II describes Satellite sensors for distinct applications. Section II is a study of Image Analysis which includes advance algorithms for preprocessing, enhancement, transformation and classification. Section IV is a brief review of Remote Sensing Applications. Section V is list of Remote sensing image analysis tools and Section VI presents conclusion.

\section{REMOTE SENSING SATELLITE IMAGERY}

Remote sensing satellite image consists of Digital Numbers [DN] which represent image features such as color, brightness, wavelength, radiated energy frequency, or picture element in the image. The smallest element on an image is called pixel. A digital image consists of pixels which are arranged in rows and columns commonly known as a raster image. The information content and dimensions of these pixels depend on the resolution of the image. Figure 1 shows various sensor resolutions.

\subsection{Spatial Resolution}

The detail of an image depends on spatial resolution of the sensor. If the spatial resolution is 10 meters, it means that each pixel denotes an area of $10 \mathrm{~m} / 10 \mathrm{~m}$ on the ground surface. Higher the resolution of an image, finer details is more clearly 


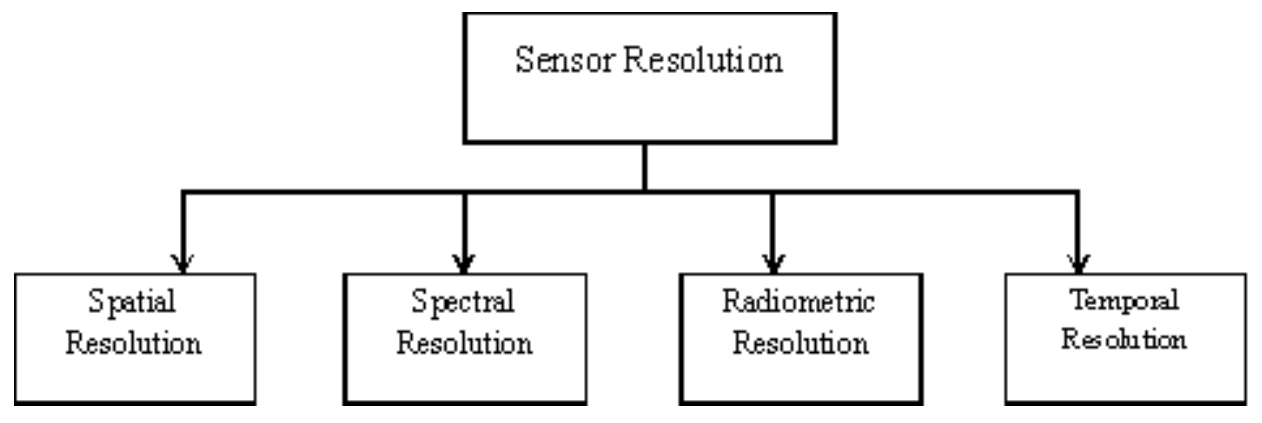

Fig 1: Satellite Sensor Resolutions

Table 1: Comparison Of Algorithms on Variable Sensor Resolution

\begin{tabular}{|c|c|c|c|c|c|}
\hline Sl.no. & Work & $\begin{array}{c}\text { Sensor } \\
\text { Resolution }\end{array}$ & Algorithm & Advantages & Disadvantages \\
\hline 1. & santi et al.,[1], 2016 & $\begin{array}{l}\text { Spatial } \\
\text { Resolution }\end{array}$ & Adhoc CLEAN & $\begin{array}{l}\text { Increased spatial resolution of } \\
\text { bistatic images }\end{array}$ & $\begin{array}{c}\text { Only for coarse range reso- } \\
\text {-lution }\end{array}$ \\
\hline 2. & Ji zhoo et al.,[3], 2016 & $\begin{array}{l}\text { Spectral } \\
\text { Resolution }\end{array}$ & $\begin{array}{l}\text { Conditional } \\
\text { Random field }\end{array}$ & $\begin{array}{c}\text { Overcomes spatial variability } \\
\text { problem }\end{array}$ & $\begin{array}{l}\text { Fail to use spatial location } \\
\text { cues information }\end{array}$ \\
\hline 3. & $\begin{array}{l}\text { Enrique no et al.[5], } \\
2013\end{array}$ & $\begin{array}{l}\text { Radiometric } \\
\text { Resolution }\end{array}$ & $\begin{array}{l}\text { Near Fiel Tech- } \\
\text { nique }\end{array}$ & $\begin{array}{l}\text { Suitable for close range } \\
\text { applications }\end{array}$ & More error rate \\
\hline 4. & H.Shyen[6], 2016 & $\begin{array}{l}\text { Temporal } \\
\text { Resolution }\end{array}$ & $\begin{array}{l}\text { Hidden Markova } \\
\text { Model }\end{array}$ & Less noise distortion & Less efficiency \\
\hline
\end{tabular}

- visible and cover less ground area. Lower the resolution of an image, details are not clearly visible but it covers larger total ground area.

Adhoc CLEAN algorithms [1] have been developed to improve the spatial resolution of bistatic images and MSAR images (Multistatic Synthetic Aperture Radar) which produces images with $1 \mathrm{~m}$ spatial resolution, however it is applicable for processing coarse range resolution.

\subsection{Spectral Resolution}

Spectral resolution of a sensor is the ability to define fine wavelength intervals in an Electromagnetic spectrum. The details of an image also depend on responses of EMR incident on an object over distinct wavelength ranges, for example, the classification of vegetation and water is usually be separated in a broad wavelength range i.e, visible and near infrared wavelengths, to distinguish different rocks needs finer wavelength range within the band to separate them. So higher the spectral resolution, narrower the wavelengths range of a particular band.

Wei et al.,[2] proposed an algorithm to fuse multiband images based on linear observation model and linear spectral mixture model which includes high spatial resolution and low spectral resolution images. It leads to increase in abundance and end member estimation parameters, but it fails to fuse spectral and spatial degradation images.

Zhao et al.,[3] integrated spatial, spectral and spatial location cues by using CRF model (Conditional Random Field) to produce high resolution remote sensing image. The proposed method solves spatial variability problem, yet it fails to use rich information available in spatial location cues.

The problem of estimating atmospheric parameters such as temperature and water vapor content in hyper spectral imagery is addressed by Ahlberg et al.,[4] using noise models; the atmospheric parameters (signature) were generated using
10- 20 spectral bands at moderate noise level but experiment fails to produce quantitative results.

\subsection{Radiometric Resolution}

Radiometric resolution enables us to recognize high and low level contrast objects in an image. Radiometric resolution describes the information about image brightness, contrast, illumination variations and other details of an image.

The study of radiometric sensitivity for 1D SA (Synthetic Aperture) and 2D SA images are discussed by Nova et al.,[5] $\mathrm{NF}$ technique (Near-Field) that produces distortion coefficients based on DCF's radiometric sensitivity with respect to close range screening applications. The disadvantage is that error rate is high for low resolution image.

\subsection{Temporal Resolution}

The time taken by a satellite to revisit the same area with same viewing angle is referred as absolute temporal resolution.

It refers to the length of time it takes for a satellite to complete one entire orbit cycle. Temporal data plays a very important role in remote sensing applications like monitoring vegetation changes, flood occurrence, deforestation, urban development etc., Spectral resolution varies with time and is identified by multi temporal imagery. Temporal resolution depends on many factors like satellite sensor capability, latitude and swath overlap.

The integration of spatial, spectral and temporal resolution [6] using hidden markov model, PSM/MS model and filtering methods are used to obtain high spatial-temporal - spectral resolution fused data. The main advantage is low noise distortion in the obtained fused image but its efficiency is low.

The spatio-temporal fusion has been done Rao et al.,[7] using high-pass frequency modulation and edge primitive 
techniques to produce high synthetic fused image of LISS III at $23.5 \mathrm{~m}$ spatial resolution and five day temporal resolution image, that overcomes computational complexity. Table I shows the comparison of algorithms to improve sensor resolutions.

\section{SATELLITE SENSORS}

Satellites provide remote sensing imagery which are commonly used today. The unique characteristics of Satellites make them particularly useful for remote sensing of the Earth's surface.

\subsection{Thermal Sensors}

To measure the surface temperature and thermal properties of a target object on the ground surface, thermal sensors are used that detects the reflected radiation from the target object.

Bian et al., [8] thermal properties of surface objects namely leaves, shaded soil and sunlit soil using multi-angle infrared airborne thermal sensors. Arbitrarily Inclined Leaves (4SAIL) model and modified FR97 model improve the precision rate in retrieving the thermal properties of these three components.

\subsection{Airborne and Space-borne Sensors}

Airborne remote sensing are one time operations. Here, sensors are mounted on aircrafts that provides images with high spatial resolution but it covers less ground area.

Space-borne remote sensing provides continuous monitoring of earth's surface. Here, the sensors are placed on space shuttles or satellites. It covers larger earth's surface with less spatial resolution.

Microwave remote sensing consists of both active and passive sensors. Longer wavelength microwave radiation penetrates in variable environmental conditions. While, shorter microwave radiation are use in the study of soil engineering.

Radar is an electromagnetic distance measuring device. The transmitter of the radar generates short microwave pulse and antenna focuses pulses into beam. The radar beam is transmitted to the earth station and hits on the object at right angle; the reflected energy from the object is recorded back within the illumination beam. As the sensor platform moves forward, recording and processing of backscattered signal builds up a two-dimensional image of the surface. Image appearance varies depending on the surface roughness, surface geometry and electrical properties of target. The radar imagery is used in oceanographic studies to detect and track ship, identify the direction of wind, monitoring natural disasters, to create DEM (Digital Elevation Model), land cover/land use classification, agriculture and forest monitoring. The process of radar beam interaction with earth's surface objects creates two dimensional image. The radar imagery is used to identify the direction of wind, oceanographic studies to detect and track ship, to create DEM (Digital Elevation Model), land cover/land use classification, monitoring natural disasters etc.

Dutta et al.,[9] Categorize vegetation canopy based on leaves area per density ( LAD) in dense forest using airborne LIDAR remote sensing imagery and hyper spectral imagery based on Pit-Free Canopy Height Model(PFCHM) and crown segmentation model. Accurate results are obtained for single dataset, yet it needs to be validate for other datasets. Radar imaging is used to detect the moving objects behind wall and to map the building interiors using GPOF (Generalized pencil of Function) algorithm. Object Point Spread Function (OPSF) technique is used to remove the ghost image of moving object which also gives 360 degree view of imaging area using omnidirectional antenna and it greatly improves the range resolution [10].

The coastal tide components in nearshore water using Xband marine radar imagery is estimated by Chen et al.,[11] based on the wave height modulation. Self- adaption model is used to remove poor quality data and type I Chebyshev bandpass filter to derive the phase and amplitude of tidal wave to predict information on coastal tide. The proposed method gives better results than OTPS model (The Oregon state university Tidal Prediction Software). The accuracy in prediction is low as the coastal waves vary with external parameters like wind. The sea surface current velocity is retrieved from X-band marine radar imagery using ILS-NSPPCS algorithms (Iterative Least Square- Normalized Scalar Product- Polar Current Shell); this approach produces accurate retrieval rate than traditional method [12].

\section{IMAGE ANALYSIS}

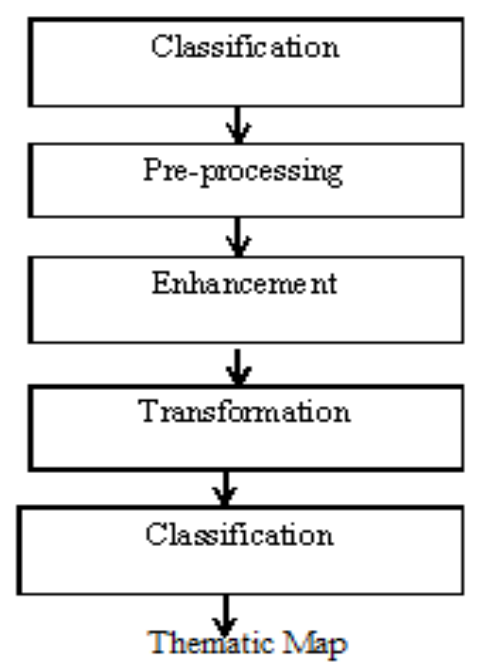

Fig 2: Remote Sensing Analysis

In order to make good use of remote sensing data, we must be able to extract meaningful information from the image by applying proper processing techniques. Remote sensing images can also be represented in a computer as arrays of pixels, with each pixel corresponding to a digital number [DN], represents the brightness level of that pixel in an image. Figure 2 is a general architecture of remote sensing image analysis.

Image analysis systems can be categorized into following:

(i) Pre-processing

(ii) Image Enhancement

(iii) Image Transformation

(iv) Image Classification and Analysis

\subsection{Pre-processing}

Pre-processing functions involves the operations required prior to the main data analysis and consists of processes aimed at geometric correction, radiometric correction and atmospheric corrections to improve the ability to interpret the image components qualitatively and quantitatively. These process correct the data for sensor irregularities and removing (radiometric corrections) unwanted sensor distortion or atmospheric noise. 


\subsubsection{Geometric Corrections}

It includes correcting the geometric distortions due to sensorEarth geometry variations and conversion of the data to real world coordinates (Geo- Referencing). When image data are recorded by satellite sensor, it contains errors in geometry. An image geometry error arises mainly due to the relative motions of the platform, its scanners and the earth. Nonidealities in the sensors, the curvature of the earth and uncontrolled variations in the position and altitude of the remote sensing platform can all lead to geometric errors of varying degrees.

Correcting the geometric errors in Nighttime Lights Time series image (NLT) have been done by Zhao et al., [13]] using GDP growth rates, cross correlation between reference image and candidate image enables to find error DN value. The population corrected NLT is used to produce accurate results but it generates uncertain errors on corrected $\mathrm{DN}$ values in nighttime light images.

Landsat MSS L1G ( Multispectral Scanners Landsat 1 Generation) images [14] are used to analyze multi-temporal changes and should be free from geometric distortions. DEM (Digital Elevation Model) and MSS sensor parameters are used for ortho-rectification and geo-referencing to improve the accuracy of geometric corrections, proposed method is evaluated quantitatively and has high accuracy.

Sola et al., [15] deal with a speedy procedure for the geometric correction of hyperspectral MIVIS images using Polynomial Model (PM) and the model of Rational Functions (RFM). The main aim is to get an effective geometric correction of MIVIS images by way of an optimal compromise of result precision and elaboration time and can be applied for large areas. The aim has been achieved by overlapping the MIVIS images and their themes with highresolution images. The main advantage of proposed method is to effectively monitor the environmental damage.

\subsubsection{Radiometric Corrections}

The reflected electro-magnetic energy are observed by a sensor, due to sun's azimuth and atmospheric conditions the observed energy does not match with the energy emitted from the same object. Therefore, in order to obtain the real irradiance or reflectance, these radiometric distortions must be corrected. A radiometric correction plays a major role in producing error free thematic map for biomass applications, to achieve this objective Simard et al.,[16] proposed holomorphic calibration technique and heteromorphic calibration techniques to correct radiometry for terrain topography and canopy variations due to reflectivity. It performs better than cosine generation technique but accuracy is low when azimuth slope is considered.

Radiometric correction model [17] is developed based on FMask code and Tanre's formulation to detect clouds and cloud shadow of Aerosol Robotic Network sun photometer data. It produces a result that is free from overlapping redundant pixels and achieves accuracy of 98.8 percent. The correction accuracy is still low for medium resolution data.

The radiometric stability in MODIS image to retrieve aqua information from ocean using Nearly Simultaneous Nadir Overpass (NSNO) technique proposed by David et al., [18], achieves more stability for deep convective cloud (DCC) invariant earth targets but fails to stabilize radiometric errors for data from different sensors.

\subsubsection{Atmospheric Corrections}

Solar radiation is absorbed or scattered by the atmosphere when it is transmitting to the earth. In the same manner the reflected energy from the target is absorbed or scattered by the atmosphere before it reaches a sensor. Sensors receive the reflected radiation from the target and also receive scattered radiation from the target is called path radiance.

The atmospheric corrections for HICO (Hyperspectral Imager for the Coastal Ocean) images are difficult because of outpouring spectral distribution of radiance from the coastal water (ocean).

Lewis et al., [19] proposed cloud-shadow atmospheric correction method, which corrects the pixels with similar optical properties such as cloud pixels, shadow pixels and sunlit pixels. This approach solves adjacency error between bright and dark objects but the success rate is low as it depends on the selection of appropriate cloud.

SAR (Synthetic Aperture Radar) [20] image has less capability in measuring ground deformation information because of Atmospheric Phase Delay Effects (APDE). Atmosphere corrected PSI model along with Advance Weather Research and Forecasting model (AWRF) overcomes baseline errors, phase noise and DEM errors in time series SAR image. This method is more suitable in studying volcanic deformation and rarely shows unexpected errors on temporal data.

Brian et al.,[21] introduced Real-time automatic atmospheric correction method on Airborne Visible/Infrared Imaging Spectrometer (AVIRIS-NG). It uses ATmospheric REMoval algorithm which reduces time complexity but automatic selection of aerosol model is still an open research problem. The preprocessing algorithms are listed in Table II.

\subsection{Enhancement}

To make image easier for visual interpretation Enhancements are used. The advantage of digital imagery is that it allows us to manipulate the digital pixel values in an image.

Although radiometric corrections for illumination, atmospheric influences, and sensor characteristics may be done prior to distribution of data to the user, the image may still not be optimized for visual interpretation.

Image Enhancement methods are of four types

(i) Radiometric Enhancement

(ii) Spatial Enhancement

(iii) Spectral Enhancement

(iv) Geometric Enhancement.

\subsubsection{Radiometric Enhancement.}

Radiometric enhancements are techniques that improve contrast between certain features of earth surface by altering the screen colors assigned to specific ranges of pixel values.

Lisani et al., [22] applied tone-mapping algorithms to the satellite images to enhance bright and shadow regions. The algorithm improves $\mathrm{K}$ - coefficient so that accuracy increases and reveals minor details in shadow but manual selection of the dark zone reduces its efficiency.

Radiometric enhancement for regions covering atmospheric products such as aerosol optical depth, perceptible water, and total ozone for KOMPSAT-3 has been developed by Yeom et al.,[23] using radiative transfer model. it is observed that the 
anisotropy factor(ANIF) showed negligible BRDF effects (Bidirectional Reflection Distribution Function); hence better enhancement is achieved but still it depends on swath overlap.

An automatic radiometric normalization for mutitemporal time series data of ETM images using Iterative Slow Feature Analysis (ISFA) was proposed by Zhang et al.,[24] that shows improved normalization coefficient NR with high efficiency but mosaicking with all kinds of images is still an open challenge.

\subsubsection{Spatial Enhancement:}

The spatial resolution of a remote sensing system is characterized either by (i) line pairs per millimeter, for airborne imagery that is typically employed when dealing with analogue or hardcopy prints or (ii) the pixel (picture element), the basic unit or building block of digital imagery. Spatial enhancement deals largely with spatial frequency and modifies pixel values based on the values of its neighbor pixels. Focal analysis, Convolution filter, Resolution Merges, Non- Directional Edge Enhancement using Laplacian filter, Texture Filters (occurence and co-occurence), Statistical Filtering and Adaptive filtering are used to enhance spatial features traditionally. Spatial resolution enhancement in bright regions of microwave remote sensing using conjugate gradient method (CG) [25]. It takes low processing and produced high reconstruction accuracy compared to Landweber method but results in low accuracy for duality maps Reconstruction technique based on 2-D truncated singular value decomposition (TSVD) [26] method to enhance spatial features reduces the noise levels (RMSE and $r$ values).

Table 2: Comparison of Algorithms on Enhancement Methods

\begin{tabular}{|c|c|c|c|c|c|}
\hline Sl.no. & Work & Algorithm & $\begin{array}{c}\text { Enhancement } \\
\text { Methods }\end{array}$ & Advantages & Disadvantages \\
\hline 1. & $\begin{array}{c}\text { min yeom } \\
\text { et al.,[23], } \\
2016\end{array}$ & $\begin{array}{c}\text { Radiometric } \\
\text { Enhancement }\end{array}$ & $\begin{array}{c}\text { Radiative } \\
\text { Transfer } \\
\text { Model }\end{array}$ & Negligible BRDF effect & Depends on swath overlap \\
\hline 2. & $\begin{array}{c}\text { Flavia lenti et al.,[26], } \\
2014\end{array}$ & $\begin{array}{c}\text { Spatial } \\
\text { Enhancement }\end{array}$ & TSVD & Reduced noise level & $\begin{array}{c}\text { Issue in selection of trunca- } \\
\text { tion parameter }\end{array}$ \\
\hline 3. & $\begin{array}{c}\text { Mohamed } \\
\text { aimebendani et } \\
\text { al.,[28],2014 }\end{array}$ & $\begin{array}{c}\text { Spectral } \\
\text { Enhancement }\end{array}$ & $\begin{array}{c}\text { Spectral } \\
\text { unmixing } \\
\text { algorithm }\end{array}$ & Low spectral distortion & $\begin{array}{c}\text { fails to fuse different reso- } \\
\text { lution images }\end{array}$ \\
\hline 4. & $\begin{array}{c}\text { Leonardo correr } \\
\text { et } \\
\text { al.,[34], 2016 }\end{array}$ & $\begin{array}{c}\text { Hidden } \\
\text { Eeometric } \\
\text { Enhancement }\end{array}$ & Markova & $\begin{array}{c}\text { Model(HMM) } \\
\text { efficiency }\end{array}$ & $\begin{array}{c}\text { Suitable only for low SNR } \\
\text { images. }\end{array}$ \\
\hline
\end{tabular}

It is robust and is compatible for hardware implementation. It is constrained by the selection of truncation parameter and kernel must be a $2 \mathrm{D}$ tensor.

A deconvolution-based model [27] has been developed to enhance the spatial features of Soil Moisture and Ocean Salinity (SMOS) data. Six different decovolution based algorithms such as SEPS, Wiener model, Wiener-War model, CLS model, CLS- WaRD model and CLS-WaRD model are applied to original synthetic image in frequency domain. It is observed that Wiener-War model results in low error rate and better enhancement rate for sea pixels and lower enhancement rate for soil pixels.

\subsubsection{Spectral Enhancement:}

Spectral enhancement is the process of creating new spectral data from available bands. New data is created on a pixel-bypixel basis by applying an operation (e.g., subtraction, division) to corresponding pixels in the existing bands Spectral Ratios and Indices and Tasseled Cap are important functions in spectral enhancement process.

Spectral resolution enhancement in multispectral images (MS) using spectral unmixing algorithm [28] reconstructs spectral as well as spatial features of MS images. Peak SNR (PSNR) and Spectral Angle Mapper (SAM) parameters describe the reconstruction quality of MS images. The algorithm shows less SAM value and hence lower spectral distortion is achieved. It fails to fuse different resolution images.

Kowkabi et al., [29] to accurately retrieve the end member class by enhancing the spectral information of satellite imagery. Clustering and over segmentation-based preprocessing (COPP) has been developed by incorporating spectral and spatial information that identifies spatially homogenous zones with the great spectral purity score. Thus it degrades local spectral variability and noise power improved computational efficiency. The proposed algorithm does not presume the presence of pure pixels.

Spectral unmixing is an important research topic in the field of remote sensing, that aims to unmix the spectral signature of different classes by enhancing the spectral features in the observed scene. Drumetz et al.,[30] developed Extended Linear Mixture Model (ELMM), that allows a pixel wise spatially coherent local variation of the end members, leading to scaled versions of reference endmembers. The scaling parameter estimates the amount of spectral variability in synthetic data and can be corrected using physical model. It gives good enhancement results for endmember class than Constrained.Least Squares Unmixing (CLSU) algorithm. It is less accurate result for aerial images like Lidar.

Kraft et al., [31] studied differences between Google earth imagery and satellite imagery to classify different classes on the earth surface.

\subsubsection{Geometric Enhancement:}

Geometric detail in an image is modified for better vision and for further processing. Geometric enhancement is characterized by its neighboring pixel. The new pixel value which is calculated from the brightness pixels of set of surrounding pixel leads to variations in observed geometric detail. Geometric enhancement in remote sensing is generally related to smoothing, edge detection and enhancement, line 
detection and enhancement. Edge and line enhancements leads to image sharpening.

Extraction of road network in urban area is necessary for proper urban planning and is an active research topic in remote sensing and geoscience. It basically involves geometric information processing like edge enhancement, smoothing and filtering.

Zang et al.,[32] developed a task-oriented enhancing technique for extracting road networks from satellite images, utilizes adaptive smoothing scheme to remove the noise or heavy texture. It sharpens the edges of road by means of anisotropic shock filter. It yields high result in terms of correctness, completeness and efficiency compared to RTV (Relative Total Variation) and RCS (Random Conditional Segmentation) methods.

The ship detection in Terra-SAR [33] using ellipse fitting and the gradient vector flow $(\mathrm{GVF})$ method where resulting values are fed to snake model to accurately detect the target ship. The Error Rate (ER) for length and width of ship are negligible and suitable for real time military applications. Radargrams are the unique instruments to investigate both terrestrial and space applications.

Carrer et al.,[34] proposed a novel preprocessing method for denoising radar acquisition image and enhancing the geometric features of radar data to detect the subsurface layers. The pixel intensity and depth parameters were mapped to Hidden Markov Model (HMM) for denoising and enhancement of radar image, Viterbi algorithm is used for detecting the subsurface layers. The main advantage is in terms of computational complexity as VA follows divide and conquer procedure. It is suitable only for low SNR images.

Rizkinia et al., [35] proposed DWT based method on to improve the quality of satellite images. DWT decompose the input image into different sub bands and threshold decomposition method is applied to identify the edges. The edges are sharpened by using morphological filters. It works well for sharpening and reducing the distortion of an image but the drawback is that it produce artifacts. A comparison of different enhancement algorithms is depicted in Table III.

\subsection{Transformation}

Image transformations typically involve the manipulation of multiple bands of data, whether from a single multispectral image or from two or more images of the same area acquired at different times (i.e. multitemporal image data). Either way, image transformations generate new images from two or more sources that highlight particular features or properties of interest, better than the original input images. Image transformation includes basic arithmetic operations like band Arithmetic operations are performed on two or more coregistered images of same geographical area. They may have different spectral band from a single multispectral data or it may have individual band of different time series data set.

Addition operation on a pair of images is performed to reduce the noise component. Subtraction operation on a pair of images is performed to find differences between those images i,e., to detect changes. Image multiplication is usually employed to extract the region of interest; it involves masking binary image with that of real image. Division or rationing operation is widely used in images of ecological, geological and agricultural applications. This operation is used to find the magnitude of differences between the spectral bands. These differences may be symptomatic of particular land cover type. Band/ Spectral Ratioing, Principal Components Analysis
(PCA) and NDVI are image transformation functions in remote sensing which has been used to monitor vegetation conditions on continental and global scales. Multisensor image fusion is an extended application of image transformation functions.

Palsson, et al., [36] proposed a method to fuse two panchromatic images, based on Principal Components Analysis (PCA), it is a statistical technique this can converts multivariate data with correlated variables into uncorrelated variables.

The new variables are obtained as linear combinations of the existing variables. In the fusion process, PCA method generates uncorrelated images. The first principal component is replaced with the panchromatic band which has higher spatial resolution. Finally, the inverse PCA transformation is applied to obtain the image in the RGB colour image. Limitations of PCA image fusion method are dominant spatial information and weak colour information which is often a Problem.

Ancuti, et al., [37] proposed a method for multi-focus image fusion based on discrete wavelet transform. Source images are decomposed by DWT method; two different window based fusion rules are separately applied to combine both low frequency and high frequency coefficients. It achieves better visual quality and objective evaluation indexes but is not implemented on multi-sensor images.

Liu et al.,[38] discussed an approach to identify minimum NDVI ( less vegetation cover) in MODIS data, approach is based on BVI-method (Brown Vegetation Index) which is a refined masking of cloud and snow matrix. The mean difference and contaminated percentage of Green band and SWIR band is less hence is useful in identifying evergreen forests, monsoon forests, and double cropping. It is evaluated by considering eleven years' time series data.

$\mathrm{Xu}$ et al.,[39] introduced a PCA-based LMMSL (Linear Minimum Mean-Square Error) denoising model for multiplicative noise in SAR image. The proposed method is a combination of PCA, log transformation and K-means clustering to find the denoising patches based on statistical characteristics of speckle noise. The clusters of all patches are used to reconstruct noise free image. MSE (Mean Square Error) and beta value is less and hence the efficiency is better compared to the existing state of art method in terms of image detail preservation and speckle noise reduction.

Yang et al.,[40] proposed Moving Weighted Harmonic Analysis (MWHA) method to reconstruct high quality vegetation NDVI time series data (removing residual effects and noise levels) of SPOT 5. MWHA method provides moving support domain to assign the weights for all the points which makes easier in determining the frequency number. A Four-Step Process (FSP) is used to determine the changes occurring in vegetation cover. It is validated with ground truth actual growth profile of vegetation and achieves accurate result. The drawback is that it is not validated for other sensor images.

\section{IMAGE CLASSIFICATION AND ANALYSIS}

Image classification is an important part of the remote sensing, image analysis and pattern recognition. Based on the idea that different feature types on the earth's surface have a different spectral reflectance and remittance properties, their recognition is carried out through the classification process. 
In a broad sense, image classification is defined as the process of categorizing all pixels in an image or raw remotely sensed satellite data to obtain a given set of labels or land cover themes. In some instances, the classification itself may be the object of the analysis. For example, classification of landuse from remotely sensed data produces a map like image as the final product of the analysis. Hence the image classification forms an important tool for examination of the digital images.
A principal application of remotely sensed data is to create a classification map of the identifiable or meaningful features or classes of land cover types in a scene.

\subsection{Supervised Classification}

The supervised classification is the essential tool used for extracting quantitative information from remotely sensed image data. Using this method, the analyst has available sufficient known pixels to generate representative parameters for each class of interest.

Table 3: Comparison of Algorithms on Supervised Classification

\begin{tabular}{|c|c|c|c|c|}
\hline Sl.no. & Work & Algorithm & Advantages & Disadvantages \\
\hline \hline 1. & $\begin{array}{c}\text { Restaino } \\
\text { et al.,[45], } \\
2016\end{array}$ & $\begin{array}{c}\text { Gabour Filter and } \\
\text { Mean Shift algo- } \\
\text { rithm } \\
\text { classification } \\
\text { result } \\
\text { for } \\
\text { variable } \\
\text { land cover type }\end{array}$ & $\begin{array}{c}\text { Less accuracy for variable texture surface } \\
\text { computational } \\
\text { efficiency }\end{array}$ & $\begin{array}{c}\text { Less accuracy for panchromatic } \\
\text { images }\end{array}$ \\
\hline 2. & $\begin{array}{c}\text { Gueguen } \\
\text { et.,[44], }\end{array}$ & POK & $\begin{array}{c}\text { Better performance } \\
\text { than } \\
\text { unsupervised } \\
\text { technique }\end{array}$ & $\begin{array}{c}\text { Fail to model non -stationary } \\
\text { property }\end{array}$ \\
\hline
\end{tabular}

This step is called training. Once trained, the classifier is then used to attach labels to all the image pixels according to the trained parameters. The quality of a supervised classification depends on the quality of the training sites, The most commonly used supervised classification is maximum likelihood classification

(MLC).[41]

Feng et al., [42] studied GRFc algorithm and Fuzzy classifier to classify different land use and land cover classes on earth surface and found that GRFC produces highest accuracy than fuzzy classifier.

Spatial and spectral properties of worldview 2 are analyzed by Arabi et al., [43]. Polynomial fit classifier is used to classify different land use objects on the earth surface and also miss classification is found in classifying finer detailed objects of the earth surface.

POK based classification algorithm [44] classifies $30 \mathrm{~m}$ resolution data with effective computational time, but his method yields low accuracy in classifying panchromatic data.

Fusion of gabour filter and variable mean shift to segment land cover types was proposed by Restaino et al.,[45]. Accuracy is high for spectral feature classification but low accuracy for textural data.

Du Peijun et al., [46] classified texture data using KNN (KNearest Neighbor) and MLC (Maximum Likely-hood Classifier) and found that KNN produces good result than MLC.

Bruzzone et al..,[48] proposed a novel pixel-based system for the supervised classification of very high geometrical (spatial) resolution images. This system aimed at obtaining accurate and reliable maps both by preserving the geometrical details in the images and by properly considering the spatial context information. Experimental results confirmed the effectiveness of the proposed system.

Wang et al., [49] describes the extended-MRF technique to develop an operational approach, which ensures high accuracy and compatibility in image classification from the satellite images of different resolutions and varying quality. The loss of forest and urban sprawl has been revealed by the analysis which is a major problem of Atlanta's city.

Mylonas et al., [50] used graph theory concept to classify urban areas. Authors found that, method yield high classification accuracy in classifying urban areas of $1 \mathrm{~m}$ resolution data. The comparison of different supervised classification algorithms is listed in Table IV.

\subsection{Unsupervised Classification:}

Pixels are grouped based on the reflectance properties of pixels. These groupings are called clusters. The user identifies the number of clusters and bands to be generated. With this information, the image classification tool generates clusters. There are different image clustering algorithms such as Kmeans and Expectation Maximization. The unsupervised classification technique is commonly used when no sample sites exist.

Authors of [52] and [53] proposed segmentation and unsupervised classification algorithm based on clustering techniques to retrieve compound objects in high resolution multispectral data.The main advantage of this method is with respect to computational time.[54]

Mei et al.,[55] developed a classification algorithm based on cellular automata Better accuracy is achieved for all $\mathrm{c} 1$ to $\mathrm{c} 6$ bands compared to other classifiers such as multilayer 
perceptron, Naive Bayes, K-NN, and RBF network but fails to classify texture data.

Shen et al., [56] proposed a method to extract thematic information of urban vegetation such as thin grass land, forest and thick grass land from quickbird imagery using traditional segmentation algorithm and fuzzy classification method. Improved K-coefficient is achieved and the result is accurate that is validated with ground truth information [57].

Table 4: Comparison of Algorithms on Unsupervised Classification

\begin{tabular}{|c|c|c|c|c|}
\hline Sl.no. & Work & Algorithm & Advantages & Disadvantages \\
\hline 1. & Xiao et al.,[52], 2016 & $\begin{array}{l}\text { Back } \\
\text { Propogation } \\
\text { and K-means }\end{array}$ & $\begin{array}{l}\text { High } \\
\text { accuracy } \\
\text { classification }\end{array}$ & Valid only for single database \\
\hline 2. & $\begin{array}{r}\text { Tiancan Mei } \\
\text { et al.,[55], } 2015\end{array}$ & $\begin{array}{r}\text { Cellular } \\
\text { Automata }\end{array}$ & $\begin{array}{l}\text { Reduced } \\
\text { noise } \\
\text { level }\end{array}$ & Failed to classify texture data \\
\hline 3. & Angel et al.,[51], 2013 & Linear filters & $\begin{array}{l}\text { Does not } \\
\text { Require prior } \\
\text { segmentation }\end{array}$ & $\begin{array}{l}\text { Improper projection for non lin- } \\
\text { ear information }\end{array}$ \\
\hline 4. & $\begin{array}{c}\text { Zhang } \\
\text { et al.,[64], 2015 }\end{array}$ & $\begin{array}{l}\text { Clustering technique } \\
\text { on 3D time } \\
\text { Series data }\end{array}$ & $\begin{array}{l}\text { Identifies dynamic } \\
\text { changes in land use } \\
\text { and land cover, }\end{array}$ & - \\
\hline
\end{tabular}

Gillis et al., [58] observe that the Mahalanobis classifier outperforms even advanced classifiers for land use/land cover. This is an accurate and simple classifier. It shows the importance of considering the data set - classifier relationship for successful image classification.

Solaiman et al., [59] have done comparative study of clustering algorithms. The study shows that DBSCAN is better than K-means and SOM in discovering non-convex cluster. SOM and K-means used to extract convex cluster. When the clusters are of hyper spherical or convex shape and well separated large dataset, K-means and SOM is preferable as they are faster [60].

Angel et al.,[61] presented a new method for remote sensing image segmentation based on spectral and texture information. Linear filters are used to enhance the spatial patterns. The proposed segmentation greatly reduce known or unknown representative feature via subspace projection. The main advantage of proposed method is that it automatically selects proper scales, which does not require segmentation at multiple scale levels. The disadvantage is that improper projection is found for nonlinear finer details.

Sen et al.,[62] proposed an automatic method for deriving Digital Terrain Models (DTM) from Digital Surface Models (DSM). The DTM generation is formalized here as the minimization of a given energy which is defined by a data term and a regularization term. It filters non ground points present in the original DSM so that the final terrain surface best fits true ground points. The method introduced was tested on a vegetated and hilly area and compared with ground truth and shows good geometric accuracy and robustness [63] [60].

Zhang et al., [64] proposed a clustering technique on 3D time series data for land use analysis such as identification of forest, urban areas, and water as well as for meteorological applications. This approach was suitable to accomplish the temporal analysis of land use. The main application of proposed work is to identify dynamic changes in land use and land cover. The comparison of different unsupervised classification algorithms are listed in Table V.

\subsection{Object-Oriented Classification:}

The object-oriented classification method suitable for medium to high resolution satellite imagery provides a valid alternative to traditional pixel-based classification. Object oriented classification involves segmenting an image into objects (groups of pixels). These objects have geographical features such as shape and length, and topological entities such as adjacency. These attributes make a knowledge base for the sample objects, which can be called upon in the classification process. Object-oriented classification has greater possibilities for detecting change in higher resolution imagery.

Lu et al.,[65] discuss an object oriented classification method for Dasymetric Mapping (DM) within remote sensing and census geography. This method overcomes many of the method. It allows the data to be independent from the arbitrary enumeration areas and therefore the ecological error is reduced in terms of area unit problem.

Yu, et al.,[66] improves the accuracy of HRI classification by fusing Feature Enhancement Techniques with ObjectOriented Classification Approach. It significantly improves the classification accuracy of HRI compared with other classical classification approaches.[67] [68].

Karouiet et al.,[69] proposed Reference Descriptor based 3Dmodel to detect car in high resolution aerial images. The radiometric features such as vehicle color, windshield color, and intensity of a car's shadow area are successfully detected but main disadvantage is that, it detects only one vehicle at a time.

Leitloff et al. [70] proposed model-based vehicle detection algorithm for parking lot aerial images. Algorithm successfully identifies vehicle in a parking lot based on the edge responses from the sides of a vehicle. The main disadvantage is that Performance degradation is found due to different camera angles.

Yang et al., [71] proposed unsupervised method based on multi-thresholding concept. The threshold is selected based on discriminant criterion moments in gray levels. The algorithm 
utilizes zeroth and first order cumulative moments. Segmentation accuracy is validated with ground truth

information [72].

Table 5: Comparison of Algorithms on Object-Oriented Classification

\begin{tabular}{|c|c|c|c|c|}
\hline Sl.no. & Work & Algorithm & Advantages & Disadvantages \\
\hline \hline 1. & $\begin{array}{c}\text { Karouiu et al.,[69], } \\
2014\end{array}$ & $\begin{array}{c}\text { RD based 3-D } \\
\text { model }\end{array}$ & $\begin{array}{c}\text { Accurate automatic } \\
\text { Detection of vehicle } \\
\text { on road }\end{array}$ & $\begin{array}{c}\text { Detects only one vehicle at a } \\
\text { time }\end{array}$ \\
\hline 2. & $\begin{array}{c}\text { leitloffet al.,[70], } \\
2013\end{array}$ & $\begin{array}{c}\text { Model based } \\
\text { Vehicle detection } \\
\text { algorithm }\end{array}$ & End to End analysis & Performance degradation \\
\hline 3. & $\begin{array}{c}\text { otsu et al..,[73], } \\
2014\end{array}$ & $\begin{array}{c}\text { Bimodal } \\
\text { thresholding }\end{array}$ & $\begin{array}{c}\text { Accurate boundary } \\
\text { description }\end{array}$ & Less efficiency \\
\hline 4. & $\begin{array}{c}\text { Sziranyi } \\
\text { et al., [76],2015 }\end{array}$ & Gamma map filter & $\begin{array}{c}\text { Identifies changes } \\
\text { in temporal data }\end{array}$ & Less efficiency \\
\hline \hline
\end{tabular}

Otsuet et al., [73] developed a semi-automatic image segmentation tool, which combines manual segmentation with novel automatic image segmentation algorithm. Manual segmentation is based on dropping control points and fitting cubic splines. Automatic segmentation is based on bimodal thresholding. By combining these two segmentation methods, accurate boundary descriptions are obtained with much less effort but it less efficient as there is more manual interpretation.

Moser et al.,[74] proposed unsupervised hierarchical image set clustering based on discrete and continuous image models. The continuous image modeling is based on a mixture of Gaussian densities and discrete image modeling is based on information-theoretic principle and the information bottleneck principle. Images are clustered such that the mutual information between the clusters and the image content is maximally preserved, which result in efficient image search and retrieval. Detecting geographical changes over a given area and generation of Digital Elevation Model (DEM) is the extended process after image classification.

Baraldi et al., [75] proposed change detection algorithm on multi-temporal SAR image to monitor the changes occur over annual cycle, based on thresholding of the cumulative histogram. to reduce the speckle noise $5 / 5$ mean filter is used. Proposed method shows accurate change detection result and is validated with ground truth information.

Sziranyi et al., [76] proposed a method to identify changes in temporal data based on block averaging, Gamma MAP filter and image segmentation. Changes are identified based on log ratio thresholding method. The main disadvantage is that the efficiency is low as they use manual thresholding to perform segmentation [77].

Berthier et al.,[78] proposed multilayer fusion model for adaptive segmentation and detecting changes in terrestrial areas of optical remote sensing image series. The method applies unsupervised or partly supervised clustering on a fused-image series by using cross-layer similarity measure, followed by multilayer Markov random field segmentation. The resulting label map is applied for the automatic training of single layers. After the segmentation of each single layer separately, changes are detected between single label maps. The significant benefit of the proposed method is numerically validated on remotely sensed image series with ground-truth data [79].
Jaehong et al.,[80] proposed a method to detect and investigate of 3-D terrain changes using Digital Elevation Models (DEMs). Stereoscopic push broom is applied to measure the ground deformation generated by a swarm of earthquakes. The algorithm successfully detects the horizontal displacements and DEM differences with about 0.1-pixel accuracy.

Guoying et al.,[81] proposed a method for quick assessing large-scale DEM landslides from high resolution SPOT-5 image. The main application of proposed method is that, it is used for damage assessments in terms of depth and volume of larg-scale landslides.

The semantic segmentation and contextual properties of different classes [82] automatically performs segmentation and classification of urban land cover type of the city of Rostock Germany, QuickBird-image and LiDAR data. Kcoefficient is more compared to CTF, TF, MRF, and SVM. The classification accuracy and efficiency is high. However it cannot utilize multiple HLC maps or multiple historical remotely sensed images at the same time. The comparison of different object-oriented classification algorithms are listed in table VI.

\section{APPLICATIONS}

The main applications of remote sensing includes Agriculture, Forestry, Geology, Hydrology, Sea ice, land cover Mapping, Oceans and Coastal [83].

\subsection{Agriculture}

To examine the health of crops, airborne images are used. It also includes monitoring farming practices [84].

\subsection{Forestry}

Forestry applications of remote sensing include environmental monitoring, commercial forestry and survey mapping To meet the objectives set by national forest and environmental departments, remote sensing is used. It includes updating forest cover, measuring biophysical characteristics. Commercial forestry applications such as monitoring vegetation density and measuring biomass parameters. It also includes monitoring forests health, quantity and diversity [85].

\subsection{Geology}

Remote sensing is a basic tool for mapping geological features such as Structural mapping, lithological mapping and 
rock mapping. It is also used to extract the information about land surface and it composition.

\subsection{Hydrology}

The active sensing capabilities of Radar imaging helps in hydrological studies. It includes mapping drainage basin, flood mapping and modeling of watershed and irrigation. It also provides us to estimate soil moisture content, snow thickness and equivalency of snow-water [86].

\subsection{Sea Ice}

Remote sensing data helps to track huge navigable cracks in the sea. It can be used to estimate the concentrate if ice, ice type, age, motion, ice-berge detection and tracking these parameters help in meteorological and global change research.

\subsection{Land Cover and Land Use}

Remote sensing techniques allows mapping of land use and land cover of earth's surface. A prior knowledge of land use and land cover helps in managing natural resources, protecting wildlife, monitoring agriculture and urban activities [87].

\subsection{Mapping}

Radar data is used for mapping which is a basic information for all remote sensing applications. It mainly includes Digital Elevation Model (DEM's) which gives the slope information of earth's surface and topographic mapping or thematic mapping.

\subsection{Oceans and Coastal Mapping}

The dynamic changes in the ocean and coastal region can be monitored and mapped using remote sensing techniques that mainly includes storm forecasting and ocean pattern recognition [88].

\section{REMOTE SENSING IMAGE ANALYSIS TOOLS}

\subsection{GRASS}

Geographic Resources Analysis Support System (GRASS) is used to perform

(i) Image Classification (ii) Radiometric Corrections (iii) Principle Component Analysis (iv) Edge Detection (v) 3D analysis (vi) Geo-statistical analysis (v) Filtering options. Another important feature of GRASS is the LiDAR processing and analysis. It provides to filter LiDAR points and to generate DEMs [89].

\subsection{PolSARPro}

This software mainly handles SAR data (TerraSARX, ALOSPALSAR, ENVISAT-ASAR and RADARSAT-2). PolSARPro is used to perform (i) Conversion (ii) Importing (iii) Decompositions (iv) Filtering (v) InSAR processing and calibration. Another important feature of this software is the graph processing framework and provides automate workflow [90].

\subsection{ArcGIS}

ArcGIS is a Geographic Information System (GIS) for working with maps and geographic information.

ArcGIS is used to perform (i) Mapping (ii) Analyzing mapped information (iii) Compiling geographic data (iv)Managing geographic information in a database (v) compiling geographic data [91].

\subsection{QGIS: Quantum GIS}

QGIS is open source GIS software available for free.

QGIS is used to perform

(i) Analyze spatial data (ii) Visualize Spatial data (iii) Interpret and understand spatial data (iv) Map algebra (v) Hydrologic modeling (vi) Surface interpolation and slope analysis. Plugins are the important features of QGIS [92][93].

\subsection{ERDAS IMAGINE}

ERDAS IMAGINE is a raster-based software package. It is designed mainly to extract information from imagery. ERDAS IMAGINE is used to perform (i) Image Ortho-rectification (ii) Re-projection (iii) classification and interpretation (iv) Mosaicking. The important feature is, it provides user to to analyze image data and present it in formats ranging from printed maps to 3D models [94].

\section{REFERENCES}

[1] F. Santi, M. Bucciarelli, D. Pastina, M. Antoniou, and M. Cherniakov, "Spatial Resolution Improvement in GNSSBased SAR using Multistatic Acquisitions and Feature Extraction," IEEE Transactions on Geoscience and Remote Sensing, vol. 54, no. 10, pp. 6217-6231, 2016.

[2] Q. Wei, J. Bioucas-Dias, N. Dobigeon, and J.-Y. Tourneret, "Hyperspectral and Multispectral Image Fusion based on a Sparse Representation," IEEE Transactions on Geoscience and Remote Sensing, vol. 53, no. 7, pp. 3658-3668, 2015.

[3] J. Zhao, Y. Zhong, H. Shu, and L. Zhang, "HighResolution Image Classification Integrating SpectralSpatial-Location Cues by Conditional Random Fields," IEEE Transactions on Image Processing, vol. 25, no. 9, pp. 4033-4045, 2016.

[4] J. Ahlberg, "Optimizing Object, Atmosphere, and Sensor Parameters in Thermal Hyperspectral Imagery," IEEE Transactions on Geoscience and Remote Sensing, 2016.

[5] E. Nova, J. Romeu, F. Torres, M. Pablos, J. M. Riera, A. Broquetas, and 1. Jofre, "Radiometric and Spatial Resolution Constraints in Mmillimeter-Wave CloseRange Passive Screener Systems," IEEE Transactions on Geoscience and Remote Sensing, vol. 51, no. 4, pp. 2327-2336, 2013.

[6] H. Shen, X. Meng, and L. Zhang, "An Integrated Framework for the Spatio-Temporal-Spectral Fusion of Remote Sensing Images," IEEE Transactions on Geoscience and Remote Sensing, vol. 54, no. 12, pp. 7135-7148, 2016.

[7] J. M. Rao, C. Rao, A. S. Kumar, B. Lakshmi, and V. Dadhwal, "Spatiotemporal Data Fusion using Temporal High-Pass Modulation and Edge Primitives," IEEE Transactions on Geoscience and Remote Sensing, vol. 53, no. 11, pp. 5853-5860, 2015.

[8] Z. Bian, Q. Xiao, B. Cao, Y. Du, H. Li, H. Wang, Q. Liu, and Q. Liu, "Retrieval of Leaf, Sunlit Soil, and Shaded Soil Component Temperatures using Airborne Thermal Infrared Multiangle Observations," IEEE Transactions on Geoscience and Remote Sensing, vol. 54, no. 8, pp. 4660-4671, 2016.

[9] Y. Han, H. Kim, J. Choi, and Y. kim, “A Shape Size Index Extraction for Classification of High Resolution 
Multispectral Satellite Images," International Journal of Remote Sensing, vol. 33, no. 6, pp. 1682-1700, 2012.

[10] B. Yektakhah and K. Sarabandi, "All-Directions Through-the-Wall Radar Imaging using a Small Number of Moving Transceivers," IEEE Transactions on Geoscience and Remote Sensing, vol. 54, no. 11, pp. 6415-6428, 2016.

[11] Z. Chen, J. Pan, Y. He, and A. T. Devlin, "Estimate of Tidal Constituents in Nearshore Waters using X-Band Marine Radar Image Sequences," IEEE Transactions on Geoscience and Remote Sensing, vol. 54, no. 11, pp. 6700-6711, 2016.

[12] W. Huang, R. Carrasco, C. Shen, E. W. Gill, and J. Horstmann, "Surface Current Measurements using Xband Marine Radar with Vertical Polarization," IEEE Transactions on Geoscience and Remote Sensing, vol. 54, no. 5, pp. 2988-2997, 2016.

[13] N. Zhao, Y. Zhou, and E. L. Samson, "Correcting Incompatible DN Values and Geometric Errors in Nighttime Lights Time-Series Images," IEEE Transactions on Geoscience and Remote Sensing, vol. 53, no. 4, pp. 2039-2049, 2015.

[14] C. Devaraj and C. A. Shah, "Automated Geometric Correction of Landsat MSS L1G Imagery," IEEE Geoscience and Remote Sensing Letters, vol. 11, no. 1, pp. 347-351, 2014.

[15] R. Amin, D. Lewis, R. W. Gould, W. Hou, A. Lawson, M. Ondrusek, and R. Arnone, "Assessing the Application of Cloud-Shadow Atmospheric Correction Algorithm on HICO," IEEE Transactions on Geoscience and Remote Sensing, vol. 52, no. 5, pp. 2646-2653, 2014.

[16] J. Jung, D.-j. Kim, and S.-E. Park, "Correction of Atmospheric Phase Screen in Time Series InSAR using WRF Model for Monitoring Volcanic Activities," IEEE Transactions on Geoscience and Remote Sensing, vol. 52, no. 5, pp. 2678-2689, 2014.

[17] B. D. Bue, D. R. Thompson, M. Eastwood, R. O. Green, B.-C. Gao, D. Keymeulen, C. M. Sarture, A. S. Mazer, and H. H. Luong, "Real-Time Atmospheric Correction of AVIRIS-NG Imagery," IEEE Transactions on Geoscience and Remote Sensing, vol. 53, no. 12, pp. 6419-6428, 2015.

[18] M. Simard, B. V. Riel, M. Denbina, and S. Hensley, "Radiometric Correction of Airborne Radar Images over Forested Terrain with Topography," IEEE Transactions on Geoscience and Remote Sensing, vol. 54, no. 8, pp. 4488-4500, 2016.

[19] D. Frantz, A. R"oder, M. Stellmes, and J. Hill, “An Operational Radiometric Landsat Preprocessing Framework for Large-Area Time Series Applications," IEEE Transactions on Geoscience and Remote Sensing, vol. 54, no. 7, pp. 3928-3943, 2016.

[20] D. R. Doelling, A. Wu, X. Xiong, B. R. Scarino, R. Bhatt, C. O. Haney, D. Morstad, and A. Gopalan, "The Radiometric Stability and Scaling of Collection 6 Terraand Aqua-MODIS VIS, NIR, and SWIR spectral bands," IEEE Transactions on Geoscience and Remote Sensing, vol. 53, no. 8, pp. 4520-4535, 2015.

[21] J.-L. Lisani, J. Michel, J.-M. Morel, A. B. Petro, and C. Sbert, "An Inquiry on Contrast Enhancement Methods for Satellite Images," IEEE Transactions on Geoscience and Remote Sensing, vol. 54, no. 12, pp.7044-7054, 2016.

[22] F. Lenti, F. Nunziata, C. Estatico, and M. Migliaccio, "Conjugate Gradient Method in Hilbert and Banach Spaces to Enhance the Spatial Resolution of Radiometer Data," IEEE Transactions on Geoscience and Remote Sensing, vol. 54, no. 1, pp. 397-406, 2016.

[23] F. Lenti, F. Nunziata, M. Migliaccio, and G. Rodriguez, "Two- Dimensional TSVD to Enhance the Spatial Resolution of Radiometer Data," IEEE Transactions on Geoscience and Remote Sensing, vol. 52, no. 5, pp. 2450-2458, 2014.

[24] M. Piles, A. Camps, M. Vall-Llossera, and M. Talone, "Spatial-Resolution Enhancement of SMOS Data: A Deconvolution-based Approach," IEEE Transactions on Geoscience and Remote Sensing, vol. 47, no. 7, pp. 2182-2192, 2009.

[25] M. A. Bendoumi, M. He, and S. Mei, "Hyperspectral Image Resolution Enhancement Using High-Resolution Multispectral Image based on Spectral Unmixing," IEEE Transactions on Geoscience and Remote Sensing, vol. 52, no. 10, pp. 6574-6583, 2014.

[26] F. Kowkabi, H. Ghassemian, and A. Keshavarz, "Enhancing Hyper spectral End member Extraction using Clustering and Over segmentation- Based Preprocessing," IEEE Transactions on Geoscience and Remote Sensing, vol. 45, no. 8, pp. 4572-4583, 2015.

[27] L. Drumetz, S. Henrot, M. A. Veganzones, J. Chanussot, and C. Jutten, "Blind Hyperspectral Unmixing using an Extended Linear Mixing Model to Address Spectral Variability," IEEE Transactions on Geoscience and Remote Sensing, vol. 25, no. 8, pp. 3890-3905, 2016.

[28] Y. Zang, C. Wang, Y. Yu, L. Luo, K. Yang, and J. Li, "Joint Enhancing Filtering for Road Network Extraction," IEEE Transactions on Geoscience and Remote Sensing, 2016.

[29] X. Zhang, B. Xiong, G. Kuang, and W. Xu, "A Geometric Parameter Extraction Method of Ship Target based on an Improved Snake Model," IEEE Transactions on Geoscience and Remote Sensing, pp. 3152-3155, 2016.

[30] L. Carrer and L. Bruzzone, "Automatic Enhancement and Detection of Layering in Radar Sounder Data Based on a Local Scale Hidden Markov Model and the Viterbi Algorithm," IEEE Transactions on Geoscience and Remote Sensing, 2016.

[31] R. Liu, "Compositing the Minimum NDVI for MODIS Data," IEEE Transactions on Geoscience and Remote Sensing, 2016.

[32] L. Xu, J. Li, Y. Shu, and J. Peng, "SAR Image Denoising via Clustering- Based Principal Component Analysis," IEEE Transactions on Geoscience and Remote Sensing, vol. 52, no. 11, pp. 6858-6869, 2014

[33] G. Yang, H. Shen, L. Zhang, Z. He, and X. Li, "A Moving Weighted Harmonic Analysis Method for Reconstructing High-Quality SPOT Vegetation NDVI Time-Series Data," IEEE Transactions on Geoscience and Remote Sensing, vol. 53, no. 11, pp. 6008-6021, 2015. 
[34] G. Moser, A. De Giorgi, and S. B. Serpico, "Multiresolution Supervised Classification of Panchromatic and Multispectral Images by Markov Random Fields and Graph Cuts," IEEE Transactions on Geoscience and Remote Sensing, vol. 54, no. 9, pp. 5054-5070, 2016.

[35] R. Restaino, M. Dalla Mura, G. Vivone, and J. Chanussot, "Context- Adaptive Pansharpening based on Image Segmentation," IEEE Transactions on Geoscience and Remote Sensing, 2016.

[36] S. K. Mylonas, D. G. Stavrakoudis, J. B. Theocharis, and P. A. Mastorocostas, "Classification of Remotely Sensed Images using the Genesis Fuzzy Segmentation Algorithm," IEEE Transactions on Geoscience and Remote Sensing, vol. 53, no. 10, pp. 5352-5376, 2015.

[37] I. Walde, S. Hese, C. Berger, and C. Schmullius, "Graphbased Mapping of Urban Structure Types from HighResolution Satellite Image Objects-Case Study of the German Cities Rostock and Erfurt," IEEE Geoscience and Remote Sensing Letters, vol. 10, no. 4, pp. 932-936, 2013.

[38] E. T. Gormus, N. Canagarajah, and A. Achim, "A Novel Decision Fusion Approach to Improving Classification Accuracy of Hyperspectral Images," IEEE Transactions on Remote Sensing and Geoscience, vol. 23, no. 11, pp. 4158 4161, 2012.

[39] F. Palsson, J. R. Sveinsson, M. O. Ulfarsson, and J. A. Benediktsson, "Model-Based Fusion of Multi-and Hyperspectral Images using PCA and Wavelets," IEEE Transactions on Geoscience and Remote Sensing, vol. 53, no. 5, pp. 2652-2663, 2015.

[40] M. Rizkinia, T. Baba, K. Shirai, and M. Okuda, "Local Spectral Component Decomposition for Multi-Channel Image Denoising," IEEE Transactions on Image Processing, vol. 25, no. 7, pp. 3208-3218, 2016.

[41] R. Hecht, G. Meinel, and M. F. Buchroithner, "Estimation of Urban Green Volume based on SinglePulse LiDAR Data," IEEE Transactions on Geoscience and Remote Sensing, vol. 46, no. 11, pp. 3832-3840, 2008.

[42] F. Ling, Y. Zhang, G. M. Foody, X. Li, X. Zhang, S. Fang, W. Li, and Y. Du, "Learning-based Super resolution Land Cover Mapping," IEEE Transactions on Geoscience and Remote Sensing, vol. 54, no. 7, pp. 3794-3810, 2016.

[43] C. Xiao, M. Liu, D. Xiao, Z. Dong, and K.-L. Ma, "Fast Closed-Form Matting using a Hierarchical Data Structure," IEEE Transactions on Circuits and Systems for Video Technology, vol. 24, no. 1, pp. 49-62, 2014.

[44] M. Espinola, J. A. Piedra-Fernandez, R. Ayala, L. Iribarne, and J. Z. Wang, "Contextual and Hierarchical Classification of Satellite Images based on Cellular Automata," IEEE Transactions on Geoscience and Remote Sensing, vol. 53, no. 2, pp. 795-809, 2015.

[45] T. Mei, L. An, and Q. Li, "Supervised Segmentation of Remote Sensing Image using Reference Descriptor," IEEE Geoscience and Remote Sensing Letters, vol. 12, no. 5, pp. 938-942, 2015.

[46] Z. Lei, T. Fang, and D. Li, "Land Cover Classification for Remote Sensing Imagery using Conditional Texton
Forest with Historical Land Cover Map," IEEE Geoscience and Remote Sensing Letters, vol. 8, no. 4, pp. 720-724, 2011.

[47] V. H. Bhat, P. G. Rao, R. Abhilash, P. D. Shenoy, K. R Venugopal, and L.M Patnaik, "A Data Mining Approach for Data Generation and Analysis for Digital Forensic Application," International Journal of Engineering and Technology, vol. 2, no. 3, p. 313, 2010.

[48] L. Gueguen, "Classifying Compound Structures in Satellite Images: A Compressed Representation for Fast Queries," IEEE Transactions on Geoscience and Remote Sensing, vol. 53, no. 4, pp. 1803-1818, 2015.

[49] P. Du, J. Xia, W. Zhang, K. Tan, Y. Liu, and S. Liu, "Multiple Classifier System for Remote Sensing Image Classification: A Review," Sensors, vol. 12, no. 4, pp. 4764 4792, 2012

[50] N. Gillis, D. Kuang, and H. Park, "Hierarchical Clustering of Hyperspectral Images using Rank-Two Nonnegative Matrix Factorization," IEEE Transactions on Geoscience and Remote Sensing, vol. 53, no. 4, pp. 2066-2078, 2015.

[51] S. Kraft, U. Del Bello, M. Bouvet, M. Drusch, and J.Moreno, "FLEX: ESA's Earth Explorer 8 Candidate Mission," IEEE Transactions on Geoscience and Remote Sensing, vol. 53, no. 7, pp. 7125-7128, 2016.

[52] G. Moser, S. B. Serpico et al., "Generalized MinimumError Thresholding for Unsupervised Change Detection from SAR Amplitude Imagery," IEEE Transactions on Geoscience and Remote Sensing, vol. 44, no. 10, pp. 2972-2979, 2006.

[53] P. D. Shenoy, K. Srinivasa, K. R Venugopal, and L. M. Patnaik, "Dynamic Association Rule Mining using Genetic Algorithms," Intelligent Data Analysis, vol. 9, no. 5, pp. 439-453, 2005.

[54] B. Solaiman, L. E. Pierce, and F. T. Ulaby, "Multisensor Data Fusion using Fuzzy Concepts: Application to LandCover Classification using ERS-1/JERS-1 SAR Composites," IEEE Transactions on Geoscience and Remote Sensing, vol. 37, no. 3, pp. 1316-1326, 2013.

[55] A. Baraldi and F. Parmiggiani, "A Refined Gamma MAP SAR Speckle Filter with Improved Geometrical Adaptivity," IEEE Transactions on Geoscience and Remote Sensing, vol. 33, no. 5, pp. 1245-1257, 2015.

[56] Y. Wang, A. I. Lyapustin, J. L. Privette, J. T. Morisette, and B. Holben, "Atmospheric Correction at AERONET Locations: A New Science and Validation Data Set," IEEE Transactions on Geoscience and Remote Sensing, vol. 47, no. 8, pp. 2450-2466, 2012.

[57] T. Daniels, W. L. Smith, and S. Kireev, "Simulation of Airborne Radiometric Detection of Wake Vortices," IEEE Transactions on Geoscience and Remote Sensing, vol. 53, no. 12, pp. 6336-6343, 2015.

[58] I. Sola, M. Gonzalez-Audicana, J. Alvarez-Mozos, and J. L. Torres, "Synthetic Images for Evaluating Topographic Correction Algorithms," IEEE Transactions on Geoscience and Remote Sensing, vol. 52, no. 3, pp. 1799-1810, 2014.

[59] L. Bruzzone and L. Carlin, "A Multilevel Context-based System for Classification of Very High Spatial 
Resolution Images," IEEE transactions on Geoscience and Remote Sensing, vol. 44, no. 9, pp. 2587-2600, 2014

[60] K. Srinivasa, A. Singh, A. Thomas, K. R. Venugopal, and L. M Patnaik, "Generic Feature Extraction for Classification using Fuzzy C-means Clustering," Intelligent Data Analysis, pp. 33-38, 2005.

[61] L. Bruzzone and L. Carlin, "A Multilevel Context-Based System for Classification of Very High Spatial Resolution Images," IEEE transactions on Geoscience and Remote Sensing, vol. 44, no. 9, pp. 2587-2600, 2012.

[62] J. Feng, L. Jiao, X. Zhang, and D. Yang, "Bag-ofVisual-Words based on Clonal Selection Algorithm for SAR Image Classification," IEEE Geoscience and Remote Sensing Letters, vol. 8, no. 4, pp. 691-695, 2013.

[63] S. Bharathi, V. Shreyas, R. Anirudh, S. Sanketh, P. D. Shenoy, K.R Venugopal, and L.M Patnaik, "Performance Analysis of Segmentation Techniques for Land Cover Types using Remote Sensing Images," 2012 Annual IEEE India Conference (INDICON), pp. 775780, 2012.

[64] A. K. Shackelford and C. H. Davis, "A Hierarchical Fuzzy Classification Approach for High-Resolutionn Multispectral Data over Urban Areas," IEEE Transactions on Geoscience and Remote Sensing, vol. 41, no. 9, pp. 1920 1932, 2003.

[65] L. Zhang, X. Huang, B. Huang, and P. Li, "A Pixel Shape Index Coupled with Spectral Information for Classification of High Spatial Resolution Remotely Sensed Imagery," IEEE Transactions on Geoscience and Remote Sensing, vol. 44, no. 10, p. 2950, 2016.

[66] J. Yuan, D. Wang, and R. Li, "Remote Sensing Image Segmentation by Combining Spectral and Texture Features," IEEE Transactions on Geoscience and Remote Sensing, vol. 52, no. 1, pp. 16-24, 2014.

[67] L. Vibha, C. Hegde, P. D. Shenoy, K. R. Venugopal, and L.M Patnaik, "Dynamic Object Detection, Tracking and Counting in Video Streams for Multimedia Mining," IAENG International Journal of Computer Science, vol. 35 , no. 3, pp. 16-21, 2008.

[68] A. Ramachandra, S. Abhilash, R. KB, and K. R. Venugopal, "Feature Level Fusion based Bimodal Biometric using Transformation Domine Techniques," IOSR Journal of Computer Engineering (IOSRJCE), vol. 3, no. 3, pp. 39-46, 2012.

[69] Q. Yu, P. Gong, N. Clinton, G. Biging, M. Kelly, and D. Schirokauer, "Object-Based Detailed Vegetation Classification with Airborne High Spatial Resolution Remote Sensing Imagery," Photogrammetric Engineering Remote Sensing, vol. 72, no. 7, pp. 799$811,2014$.

[70] A. Angel, M. Hickman, P. Mirchandani, and D. Chandnani, "Methods of Analyzing Traffic Imagery Collected from Aerial Platforms," IEEE Transactions on Intelligent Transportation Systems, vol. 4, no. 2, pp. 99107, 2003.

[71] J. Goldberger, S. Gordon, and H. Greenspan, "Unsupervised Image-Set Clustering using an Information Theoretic Framework," IEEE Transactions on Image Processing, vol. 15, no. 2, pp. 449-458, 2012.
[72] H. S. Kumar, K. B Raja, K. R Venugopal, and L. M Patnaik, "Automatic Image Segmentation using Wavelets," International Journal of Computer Science and Network Security, vol. 9, no. 2, pp. 305-313, 2009.

[73] M. Celenk, "A Color Clustering Technique for Image Segmentation," Computer Vision, Graphics, and Image Processing, vol. 52, no. 2, pp. 145-170, 2014.

[74] I. Karoui, R. Fablet, J.-M. Boucher, and J.-M. Augustin, "Variational Region-based Segmentation using Multiple Texture Statistics," IEEE Transactions on Image Processing, vol. 19, no. 12, pp. 3146-3156, 2013.

[75] R. R. Muskett, C. S. Lingle, J. M. Sauber, A. S. Post, W. V. Tangborn, B. T. Rabus, and K. A. Echelmeyer, "Airborne and Spaceborne DEM and Laser AltimetryDerived Surface Elevation and Volume Changes of the Bering Glacier System, Alaska, USA, and Yukon, Canada, 1972-2006”, Journal of Glaciology, vol. 55, no. 190, pp. 316-326, 2013.

[76] E. Berthier, E. Schiefer, G. K. Clarke, B. Menounos, and F. Remy, "Contribution of Alaskan Glaciers to Sea-Level Rise Derived from Satellite Imagery," Nature Geoscience, vol. 3, no. 2, pp. 92-95, 2014.

[77] K. R Venugopal, K. Srinivasa, and L. M. Patnaik, Soft Computing for Data Mining Applications. Springer, 2009.

[78] T. Toutin, "Digital Elevation Model Generation Over Glacierized Region," Encyclopedia of Snow Ice and Glaciers, pp. 202-213, 2011.

[79] P. D. Shenoy. K. R. Venugopal, Vibha, Harshavardhan $\mathrm{L}$ and L. M. Patnaik, "Lesion Detection using Segmentation and Classification of Mammograms," Proceedings of the 25th conference on Proceedings of the 25th IASTED International Multi-Conference: artificial intelligence and applications, pp. 311-316, 2007.

[80] C. S. Fraser and H. B. Hanley, "Bias-Compensated RPCs for Sensor Orientation of High-Resolution Satellite Imagery," Photogrammetric Engineering and Remote Sensing, vol. 71, no. 8, pp. 909-915, 2015.

[81] S. Jia, G. Tang, J. Zhu, and Q. Li, "A Novel RankingBased Clustering Approach for Hyperspectral Band Selection," IEEE Transactions on Geoscience and Remote Sensing, vol. 54, no. 1, pp. 88-102, 2016.

[82] G. Baudat and F. Anouar, "Generalized Discriminant Analysis using a Kernel Approach," Neural computation, vol. 12, no. 10, pp. 2385-2404, 2015.

[83] Y. Wang, X. X. Zhu, B. Zeisl, and M. Pollefeys, "Fusing Meter- Resolution 4-D InSAR Point Clouds and Optical Images for Semantic Urban Infrastructure Monitoring," IEEE Transactions on Geoscience and Remote Sensing, 2016.

[84] R. A. Schowengerdt, Remote Sensing: Models and Methods for Image Processing. Academic press, 2006.

[85] G. Camps-Valls, D. Tuia, L. G'omez-Chova, S. Jim'enez, and J. Malo, "Remote Sensing Image Processing," Synthesis Lectures on Image, Video, and Multimedia Processing, vol. 5, no. 1, pp. 1-192, 2011.

[86] J. R. Schott, "Remote Sensing". Oxford University Press, 2007. 
[87] R. A. Schowengerdt, Techniques for Image Processing and Classifications in Remote Sensing. Academic Press, 2012.

[88] G. Cmara, R. C. M. Souza, U. M. Freitas, and J. Garrido, "SPRING: Integrating Remote Sensing and GIS by Object-Oriented Data Modelling," Computers graphics, vol. 20, no. 3, pp. 395-403, 2006.

[89] E. Pottier, L. Ferro-Famil, S. Allain, S. Cloude, I. Hajnsek, K. Papathanassiou, A. Moreira, M. Williams, A. Minchella, M. Lavalle et al., "Overview of the PolSARpro V4. 0 Software. The Open Source Toolbox for Polarimetric and Interferometric Polarimetric SAR Data Processing," 2009 IEEE International Geoscience and Remote Sensing Symposium, vol. 4, pp. IV-936, 2011.

[90] K. Johnston, J. M. Ver Hoef, K. Krivoruchko, and N. Lucas, Using ArcGIS Geostatistical Analyst. Esri Redlands, 2001, vol. 3.
[91] M. Gooch, J. Chandler, and M. Stojic, "Accuracy Assessment of Digital Elevation Models Generated using the Erdas Imagine OrthoMAX Digital Photogrammetric System," The Photogrammetric Record, vol. 16, no. 93, pp. $519-531,2010$.

[92] M. V. Martin, W. D. Goran, R. C. Lozar, J. M. Messersmith, and M. S. Ruiz, "GRASS/GIS (Geographic Resources Analysis Support System/Geographic Information System) Implementation Guide," DTIC Document, Tech. Rep., 1989.

[93] K. R Venugopal and R. Buyya, Mastering C++. Tata McGraw-Hill Education, 2013.

[94] S. Steiniger and A. J. Hunter, "Free and Open Source GIS Software for Building a Spatial Data Infrastructure," Geospatial free and open source software in the 21 st century, pp. 247-261, 2012. 